\title{
Cough Variant Asthma in Medical Outpatient Department of a Tertiary Care Hospital in Bangladesh
}

\author{
Swapna Bhattacharjee ${ }^{1}$, Shekhar Bhattacharjee ${ }^{1}$, Rukhsana Parvin ${ }^{2}$
}

\begin{abstract}
Background: Cough variant asthma (CVA) is a subset of asthma where the only symptom is chronic persistent cough. Many cases go unrecognized due to lack of proper evaluation. Response to asthma medication with features supportive of airway hypersensitivity helps in management of this disease. Objective: To find out the proportion of cough variant asthma among the patients attending medicine outpatient department of Enam Medical College, Savar, Dhaka. Materials and Methods: This cross sectional study was conducted in Enam Medical College Hospital, Savar, Dhaka over a period of two years from July 2009 to July 2011. Cough variant asthma was diagnosed mainly on clinical ground as chronic cough without wheezing, fever, weight loss, shortness of breath or sputum or any other apparent cause that persisted for more than eight weeks with absolutely normal physical examination of chest, normal chest radiography and blood count except raised eosinophil count and IgE level. Patients who met these criteria were given 2 weeks course of inhaler beclomethasone propionate and were assessed for improvement. Those who improved after steroid inhalation were categorised as having cough variant asthma. Results: Out of purposively selected 148 patients complaining only of chronic dry cough for more than eight weeks, 92 patients met the primary selection criteria for cough variant asthma. These 92 patients were given 2 weeks trial of $250 \mu \mathrm{gm}$ beclomethasone inhalation twice daily. Seventy nine patients reported almost complete recovery from chronic cough after 2 weeks and were categorized as having CVA. Thirteen patients did not improve and were not categorized as CVA. Conclusion: These findings suggest that cough variant asthma is the most common among the patients with chronic cough not due to any apparent cause. The efficacy of inhaled corticosteroid suggests that early intervention is effective in the treatment of this disease.
\end{abstract}

Key words: Cough variant asthma, Chronic cough

J Enam Med Col 2013; 3(1): 29-31

\section{Introduction}

Cough is one of the most common symptoms described by patients attending medicine outpatient clinic in general hospitals in Bangladesh. Bronchial asthma and cough variant asthma (CVA) comprise the major bulk of otherwise healthy patients complaining of chronic nonproductive cough besides other causes like post-infection post nasal drip syndrome (PNDS) and cough associated with gastroesophageal reflux disease (GERD) ${ }^{1-3}$ CVA can be considered as a subtype of bronchial asthma and about $25 \%$ of CVA patients seem to develop clinically full-blown bronchial asthma. CVA is characterized by persistent dry cough more than eight weeks without apparent cause, absence of wheeze or dyspnea, normal chest examination as well as normal chest radiography with normal blood

1. Former Professor, Department of Medicine, Enam Medical College \& Hospital, Dhaka

2. Assistant Professor, Department of Medicine, Enam Medical College \& Hospital, Savar, Dhaka

Correspondence Swapna Bhattacharjee,Email: shbhattacharjee07@yahoo.com 
count except eosinophilia. Spirometry is normal but there is bronchial hyperreactivity and cough is relieved with bronchodilator and/or steroid inhalation. ${ }^{4}$ The pathophysiology of CVA is poorly understood. It is suggested that an imbalance between the production of bronchoconstrictor leucotriene $\mathrm{C} 4$ and bronchoprotective prostaglandin E4 lipid mediators may be responsible for development of CVA.

The studies of prevalence and significance of CVA in Bangladesh are limited. We therefore have evaluated patients with chronic cough at medicine outpatient department in Enam Medical College Hospital, Savar, Dhaka to find the percentage of CVA among patients with chronic cough mostly on clinical grounds. We have used a therapeutic challenge with inhaler corticosteriod as part of diagnostic procedure instead of methacholine challenge test. ${ }^{5}$

\section{Materials and Methods}

This cross sectional study was conducted over a period of two years from July 2009 to July 2011 at the Medicine Outpatient Department (OPD) in Enam Medical College Hospital. All new patients with a complaint of cough for more than eight weeks were enrolled. Detailed history of patients including fever, sputum, hemoptysis, dyspnea, wheeze, weight loss, smoking habit, occupation was taken and drug history especially current consumption of angiotensin converting enzyme inhibitor (ACEI) and/or $\beta$ blocker was taken.

Patients with history of weight loss, fever, sputum, hemoptysis, dyspnea, and wheeze were excluded from the study. Similarly patients having smoking habit or history of working in industry or taking $\beta$ blocker or ACEI were excluded. Each patient's chest was examined carefully by two consultants separately for any crepitation or rhonchi or any other abnormal physical finding and if found were excluded. For rest of the patients, chest radiography, sputum for acid fast bacilli and gram stain, ESR, CRP, full blood count, circulating eosinophil and $\operatorname{lgE}$ level were done. Any patients having abnormality in the above tests except raised eosinophil count and IgE level were excluded. Spirometry test and methacholine challenge test could not be done due to limited facilities.
During the two years study a total number of 4792 new patients were seen in adult Medicine OPD of whom 148 patients having chronic non-productive cough for more than eight weeks were admitted. Of these patients, 92 were identified initially as having CVA on the basis of history, physical examination, and tests mentioned above. All the selected patients were found to have taken one or more full courses of antibiotic (amoxycillin, azithromycin or quinolone) and antitussive before attending OPD and so no further antibiotic or antitussive was prescribed. Rather each patient was prescribed inhaler beclomethasone proprionate $250 \mu \mathrm{g}$ twice daily (the technique of inhaler use was shown). After 2 weeks each patient was examined and enquired about response to treatment and the outcome was recorded.

\section{Results}

Out of 92 patients who were prescribed to inhale beclomethasone $250 \mu \mathrm{g}$ twice daily for 2-3 weeks, 79 patients $(85.9 \%)$ reported that their symptoms either disappeared or decreased substantially and were categorized as CVA. Remaining 13 patients (14.1\%) who did not improve were not categorized as CVA patients.

Table I: Response to beclomethasone $(\mathrm{n}=92)$

\begin{tabular}{|l|c|c|}
\hline Response to beclomethasone & Number & Percentage \\
\hline Symptoms disappeared & 79 & 85.9 \\
\hline No improvement & 13 & 14.1 \\
\hline
\end{tabular}

\section{Discussion}

CVA was first described by Glauser in $1972 .^{5}$ CVA has been defined as chronic persistent cough without wheeze or rhonchi not due to any apparent causes and relief of coughing following bronchodilator therapy or inhaled steroid but not with antibiotics or antitussives. ${ }^{6}$ Chronic cough is defined as cough lasting $>3$ weeks, but cough due to virus, mycoplasma infection and non-specific transient cough with even 8 weeks' duration can be excluded. ${ }^{7,8}$

Among total 148 healthy patients complaining dry cough $>8$ weeks in this study, 79 (54\%) were found having CVA. This finding is higher than that found in western countries as reported by Mc Graven et $\mathrm{al}^{9}(35 \%)$ and by Irwin et al ${ }^{10}$ but consistent with the 
finding reported from Japan by Shirahata et $\mathrm{al}^{11}(61 \%)$. The difference with western countries might be due to higher prevalence of GERD and PNDS in those countries where these conditions constitute around $10-27 \%$ and $29-41 \%$ of chronic cough cases respectively. ${ }^{1-3}$ The prevalence of GERD and PNDS associated chronic cough is very low in many countries including Japan. ${ }^{12}$ However, in the present study PNDS and GERD could not be investigated. Furthermore, atopic cough could not be differentiated from CVA as we prescribed steroid inhalers from the beginning, to which both atopic cough and CVA respond equally ${ }^{12}$ and we could not evaluate airway sensitivity.

CVA is one of the most common causes of chronic nonproductive cough in otherwise healthy patient. Absence of dyspnea, dry cough, normal chest findings and unremarkable chest radiography strongly suggest the diagnosis. In the context of low socioeconomic condition of our population, we can diagnose the condition by history, physical examination and simple tests and if treated early with steroid inhaler, development of asthma in future may be prevented in most of the patients. The findings of this study might be helpful for physicians as well as patients to avert unnecessary and costly investigations and medicine which may even be harmful when irritating cough becomes bothersome.

\section{References}

1. Poe RH, Harder RV, Israel RH, Kallay MC. Chronic persistent cough. Experience in diagnosis and outcome using an anatomic diagnostic protocol. Chest 1989; 95: 723-728.

2. Irwin RS, Curley FJ, French CL. Chronic cough. The spectrum and frequency of causes, key components of the diagnostic evaluation and outcome of specific therapy. Am. Rev. Respir. Dis. 1990; 141: 640-647.

3. Palombini BC, Villanova CA, Araújo E, Gastal OL, Alt DC, Stolz DP et al. A pathogenic triad in chronic cough: asthma, postnasal drip syndrome, and gastro-oesophageal reflux disease. Chest 1999; 116: 279-284.

4. Fujimara M, Ogawa H, Nishizawa Y, Nishi K. Comparison of atopic cough with cough variant asthma: is atopic cough a precursor of asthma? Thorax 2003; 58: 14-18.

5. Glauser FL. Variant asthma. Ann. Allergy Asthma Immunol. 1972; 30: 457-459.

6. Fujimara M, Sakamoto S, Matsuda T. Bronchodilator resistive cough in atopic patients: bronchial reversibility and hyperresponsiveness. Intern Med. 1992; 31: 447-452.

7. Empey DW, Laitinen LA, JaCab SL, Gold WM, Nadel JA. Mechanisms of bronchial hyperreactivity in normal subjects after upper respiratory tract infection. Am Rev. Resp. Dis. 1976; 113: 131-139.

8. Little JW, Hall WJ, Douglas RG Jr, Mudholkar GS, Speers DM, Patel K. Airway hyperreactivity and peripheral airway dysfunction in influenza A infection. Am Rev. Resp. Dis. 1978; 118: 295-303.

9. McGarvey LP, Heaney LG, Lawson JT, Johnston BT, Scally CM, Ennis $\mathrm{M}$ et al. Evaluation and outcome of patients with chronic nonproductive cough using a comprehensive diagnostic protocol. Thorax 1998; 53: 738-743.

10. Irwin RS, Carrao WM, Pratter MR. Chronic persistent cough in adult, the spectrum and frequency of causes and successful outcome of specific therapy. Am Rev. Resp. Dis. 1981; 123: 413-417.

11. Shirahata K, Fujimoto K, Arioka H, Shouda R, Kudo K, Ikeda S. Prevalence and clinical feature of cough variant asthma in a general internal medicine outpatient clinic in Japan. Respirology 2005; 10: 354-358. 